\title{
Social Insurance for Long-Term Care with Deductible and Linear Contributions
}

\author{
Justina Klimaviciute and Pierre Pestieau*
}

Received 02 February 2017; in revised form 31 July 2017; accepted 29 September 2017

With the rapid increase in long-term care (LTC) needs, the negligible role of the market, and the declining role of informal family care, one might expect that the government would take a more proactive role in the support of dependent elderly, particularly those who cannot, whatever the reason, count on assistance from their family. The purpose of this paper is to analyze the possibility of designing a sustainable public LTC scheme that would meet a widespread concern, that about going bankrupt and being unable to bequeath any saving to one's children.

Keywords: long-term care, deductible theorem, capped spending, optimal taxation

JEL classification: H 21, I 13, J 14

\section{Introduction}

Due to the aging process, the rise in long-term care needs constitutes a major challenge of the coming decades. Long-term care (LTC) concerns individuals who are no longer able to carry out basic daily activities such as eating, washing, dressing, etc. Nowadays, the number of persons in need of LTC is substantial. According to Frank (2012), in 2010 nearly 10 million Americans required ongoing help through LTC. This number is expected to grow to 15 million by 2020. Similarly, in Europe the number of persons in need of LTC is expected to grow from 27 million in 2013 to 35 million by year 2060 (see European Commission, 2015).

* Klimaviciute: Université de Liège, Liège, Belgium (justina.klimaviciute@ulg.ac.be); Pestieau: Université de Liège, Liège, Belgium (p.pestieau@ulg.ac.be). This paper is part of the special issue of FinanzArchiv/Public Finance Analysis commemorating Hans-Werner Sinn's 70th birthday. We thank the Editor Alfons Weichenrieder and two anonymous referees for their helpful comments and suggestions. We are also grateful to the participants of the 16th Journées Louis-André Gérard-Varet, the APET 2017 conference and seminars in Pisa, Strasbourg, and Paris Nanterre for useful remarks and questions. Financial support from the Belgian Science Policy Office (BELSPO) research project CRESUS is gratefully acknowledged. P. Pestieau gratefully acknowledges financial support from the Chaire "Marché des risques et création de valeur" of the FDR/SCOR.

FinanzArchiv 74 (2017), 88-108 ISSN 0015-2218 doi: $10.1628 / 001522118 \times 15084133837809$

(C) 2017 Mohr Siebeck 
The expected rise in the number of persons in need of LTC raises the question of the provision of care. As stressed by Norton (2000), about two-thirds of LTC is generally provided by informal caregivers (mainly the family, i.e., spouses, daughters, and stepdaughters). Recent figures in Frank (2012) show that about $80 \%$ of dependent individuals in the U.S. receive informal care from relatives and friends. The remaining LTC is provided formally, that is, through services that are paid for on the market. Formal care can be provided either at the dependent's home, or in an institution (care center or nursing home). Whereas LTC services do not require high skills, they are nonetheless extremely expensive. Those large costs raise the question of the funding of formal LTC. And that question will become increasingly important in the future, when it is expected that the role of informal LTC provision will decrease. According to the 2015 Aging Report (European Commission, 2015), one can foresee at the same time an increase in the needs for LTC and "a shift from informal care towards formal care-giving as typical caregivers get more involved in the labor market and the new family structures may imply less support to the older generations" (European Commission, 2015, p. 147). The implication of this is that financial risks associated with meeting LTC needs will grow and therefore the development of mechanisms for absorbing these risks will gain in importance.

Given that each person has a large probability of entering a nursing home when becoming old and given the large costs of these institutions, one would expect that private LTC insurance markets would expand, in order to insure individuals. However, although markets for private LTC insurance exist, these remain thin in most countries. According to Brown and Finkelstein (2007), only about 9 to $10 \%$ of the population at risk of facing future LTC costs has purchased a private LTC insurance in the U.S. This is the so-called long-term care insurance puzzle. For various reasons pertaining both to the demand side (myopia, denial of LTC, crowding out by the family, etc.) and to the supply side of that market (high loading factors, unattractive reimbursement rules, etc.), only a small fraction of the population buys LTC private insurance. One can thus hardly rely only on the development of private LTC insurance markets to fund the cost of LTC.

In the light of the expected decline in informal care, and of the difficulties faced by the market for private LTC insurance, one may expect that the public sector will play a more important role in the provision and funding of LTC. Nowadays, in most advanced economies, the state is involved either in the provision or in the funding of LTC services, but to an extent that varies strongly across countries. However, the involvement of the public sector in LTC is not as comprehensive and generous as it is for the funding of general health ser-

1 Pestieau and Ponthière (2012). 
vices. The LTC pillar of the welfare state remains quite thin in comparison with other pillars of the social insurance system.

Recently a number of papers have looked at the design of optimal social insurance for LTC. ${ }^{2}$ In most cases, they assume at the outset that the LTC public benefit is flat and thus not related to the severity of the dependence, nor to the amount of contributions. Those papers do not meet one of the concerns of most dependents, which is that they might incur very large costs that would force them to sell all their assets and prevent them from bequeathing any of them. This concern is not met by current LTC practices either. This concern could be dealt with by a system in which individuals' contributions to their LTC costs are capped at a certain amount after which individuals would be fully covered for all further expenditures. Such a system was proposed in the UK by the Dilnot Commission (2011). The Dilnot Commission describes the rationale for this suggestion in terms of the benefits of insurance. While only a fraction of the dependents (in their estimates around a third) would reach the proposed cap of about $£ 35,000$, everyone would benefit from knowing that if they ended up in the position of facing these costs, they would be covered, removing the fear and uncertainty of the current system (Dilnot Commission, 2011, p. 32).

We argue that this proposed formula can be justified as an efficient insurance policy, applying Arrow's (1963) theorem on insurance deductibles. This theorem goes as follows: "If an insurance company is willing to offer any insurance policy against loss desired by the buyer at a premium which depends only on the policy's actuarial value, then the policy chosen by a risk-averting buyer will take the form of $100 \%$ coverage above a deductible minimum" (Arrow, 1963). In an earlier paper, Klimaviciute and Pestieau (2017), we show that optimal social LTC insurance indeed features a deductible as long as there are loading costs. In that paper, we study a nonlinear policy allowing for the deductibles to differ between the individual types and the states of nature. In the present paper, we want to explore a more restricted policy in which the government is constrained to use linear instruments and the same deductible for all types and in both dependence states of nature. We consider thus a social insurance scheme that consists of a linear payroll tax and $100 \%$ coverage of LTC risks above a deductible. Another feature of this paper is that besides the heterogeneity in income we consider the reasonable hypothesis that there is a negative correlation between the income levels and the probability of dependence.

Before proceeding, a comment is in order. Both Dilnot Commission (2011) and our analysis are looking for the optimal design of a social insurance that would meet the concern of most individuals, namely avoiding losing all one's

2 See, e.g., Cremer et al. (2016), Cremer and Roeder (2013), Pestieau and Sato (2008). 
assets in case of a too long and too severe state of dependence. Such a scheme does not exist in the real world. Yet through a number of social assistance programs many countries offer some kind of protection against those "catastrophic" risks. The best example of this is Medicaid in the U.S., which provides LTC support to the poor and to the middle class elderly who incur a long and costly period of disability. Unfortunately, these programs are not as generous and universal as required by those risks, which are expected to grow rapidly in the next decades.

The rest of the paper is organized as follows. Section 2 presents the model. Section 3 discusses optimal public policy in the absence of private LTC insurance, and section 4 looks at the case when private insurance is available. Section 5 concludes. Some additional analysis and more technical material are provided in the appendix (section 6).

\section{The Model}

We consider a two-period model with a society consisting of $N$ types of individuals. Individuals differ in their first-period income $y_{i}(i=1, \ldots, N)^{3}$ and in their probabilities of becoming dependent in the second period. In the first period, individuals choose how to allocate their disposable income between their first-period consumption $c_{i}$ and their savings $s_{i}(i=1, \ldots, N)$ for the second period. In the second period, individuals face the risk of becoming dependent. With probability $\pi_{1 i}(i=1, \ldots, N)$, they experience a low severity level of dependence, in which case they have LTC needs (expressed in terms of costs incurred) $L_{1}$; with probability $\pi_{2 i}(i=1, \ldots, N)$, they face a heavy dependence with LTC needs $L_{2}>L_{1}$; and with probability $1-\pi_{1 i}-\pi_{2 i}$, they remain healthy. We assume that the risk of dependence is negatively correlated with individual income, i.e., $\pi_{1 j}>\pi_{1 k}$ and $\pi_{2 j}>\pi_{2 k}$ for all $j, k$ for which $y_{j}<y_{k}$. We first assume that there is no market for private LTC insurance (reflecting the fact that, as mentioned in the introduction, private LTC insurance market is in most countries very small or nonexistent), but later on, in section 4 , we also consider the case where private insurance purchases are possible.

We consider a government that introduces a public policy consisting of a linear income tax of rate $\tau$ used to finance social LTC insurance and a demogrant $A$ provided in the first period (in section 6.1 we also discuss the case without a demogrant). Most of our analysis focuses on the case of a utilitarian government, but in section 3 we also look at the case of a Rawlsian social

3 For simplicity, we do not explicitly model individual choices of labor supply and consider individual income as exogenously given. 
welfare function, which allows us to derive some deeper insights into the influence of redistributional concerns. We discuss the Rawlsian case further in section 4 . We allow for inefficiency in tax collection by assuming that a tax rate $\tau$ is associated with a quadratic $\operatorname{cost} \frac{\gamma \tau^{2}}{2}$, with $\gamma>0$. We also assume that insurance provision is not costless for the government, i.e., the government faces loading costs $\lambda>0$ that reflect, for instance, the associated administrative expenses. Following Arrow's (1963) theorem of the deductible, we consider a social LTC insurance scheme in which individuals have to pay for their LTC needs themselves up to a certain amount $D$, above which the costs are fully covered by the government. Note, however, that if LTC costs in some state of nature are lower than $D$, the government provides no insurance in that state and the individuals simply pay the entirety of their costs. We will assume that $D$ is always lower than the costs in the state of heavy dependence $\left(L_{2}\right)$, but will consider the possibility that it is higher than the costs in the state of light dependence $\left(L_{1}\right)^{4}$

Denoting by $c_{i}^{D_{1}}, c_{i}^{D_{2}}$, and $c_{i}^{I}$ the second-period individual wealth levels (net of LTC costs) in respectively the light-dependence, the heavy-dependence, and the healthy states, the expected utility of an individual $i(i=1, \ldots, N)$ can be written as follows: ${ }^{5}$

$$
\begin{aligned}
& U_{i}=u\left(c_{i}\right)+\pi_{1 i} u\left(c_{i}^{D_{1}}\right)+\pi_{2 i} u\left(c_{i}^{D_{2}}\right)+\left(1-\pi_{1 i}-\pi_{2 i}\right) u\left(c_{i}^{I}\right), \\
& \text { where } \quad c_{i}=y_{i}(1-\tau)+A-s_{i}, \\
& c_{i}^{D_{1}}= \begin{cases}s_{i}-D & \text { if } D \leq L_{1}, \\
s_{i}-L_{1} & \text { if } D>L_{1},\end{cases} \\
& c_{i}^{D_{2}}=s_{i}-D \text { and } c_{i}^{I}=s_{i} .
\end{aligned}
$$

It should be noted that we do not model individuals' bequests explicitly, but rather focus on their total second-period wealth. An alternative could be to add a joy-of-giving to the utility function, but this would not change the essence of the analysis. We therefore concentrate on individuals' total wealth, assuming implicitly that they decide how to allocate this wealth between their old-age consumption and bequests left to their children. As long as bequests are considered as normal goods, wealthier individuals will leave higher bequests. In other words, individuals want to smooth both their consumption and their bequests across the states of nature.

4 In this paper we assume that dependence occurs in the whole second period of life and that it is measured in monetary units. In a more realistic model, dependence could occur at any age and last as long as life. In that case an insurance with deductible would cover all LTC expenses beyond a given length of dependence. For this, see Drèze et al. (2016).

5 For simplicity, we assume that individuals have the same utility functions in both periods and in all states of nature. Another way would be to assume state-dependent preferences, but this makes the problem much more complicated. 
The individual choice of savings is made so as to satisfy the following firstorder condition (FOC):

$$
u^{\prime}\left(c_{i}\right)=\pi_{1 i} u^{\prime}\left(c_{i}^{D_{1}}\right)+\pi_{2 i} u^{\prime}\left(c_{i}^{D_{2}}\right)+\left(1-\pi_{1 i}-\pi_{2 i}\right) u^{\prime}\left(c_{i}^{I}\right) .
$$

Note that, in the presence of a negative correlation between individual income and the risk of dependence, the comparison of savings chosen by different individual types is generally ambiguous and depends on the differences in $y$ and on dependence probabilities. If, for instance, there are only small differences in $y$ but large differences in dependence probabilities between the types, it is possible that poorer individuals will save more than the richer ones because they have a higher risk of experiencing the states of nature with losses (LTC costs). It seems, nevertheless, that such a situation is less likely to occur and that it is more reasonable to expect differences in $y$ to be larger than differences in dependence probabilities. In what follows, we therefore assume this more reasonable scenario and consider that savings of richer individuals are higher than those of poorer ones.

In order to focus on the influence of redistributional concerns, we also make an assumption that the loading costs $\lambda$ are not too large, so that, from the pure point of view of insurance provision, insuring individuals against LTC costs (i.e., proposing $D<L_{2}$ ) is desirable. More specifically, we assume that at the point $D=L_{2}$, we have

$$
\lambda<\frac{\sum n_{i}\left[u^{\prime}\left(c_{i}^{D_{2}}\right)-u^{\prime}\left(c_{i}\right)\right]}{\sum n_{i} u^{\prime}\left(c_{i}\right)}
$$

where $n_{i}$ is the share of type $i$ individuals in the society $\left(\sum n_{i}=1\right){ }^{6}$

\section{Optimal Linear Policy without Private Insurance}

We now turn to the derivation of the optimal public policy, and we first study the case of a utilitarian government.

6 This condition is derived from the fourth term in equation (7) or the third term in equation (21). 


\subsection{Utilitarian Case}

The Lagrangian of the government's problem can be written as follows: ${ }^{7}$

$$
\begin{gathered}
\mathcal{L}=\sum n_{i}\left[u\left(y_{i}(1-\tau)+A-s_{i}\right)+\pi_{1 i} u\left(s_{i}-D\right)\right. \\
\left.+\pi_{2 i} u\left(s_{i}-D\right)+\left(1-\pi_{1 i}-\pi_{2 i}\right) u\left(s_{i}\right)\right] \\
+\mu \sum n_{i}\left[\left(1-\frac{\gamma \tau}{2}\right) \tau y_{i}-A-(1+\lambda) \pi_{1 i}\left(L_{1}-D\right)\right. \\
\left.-(1+\lambda) \pi_{2 i}\left(L_{2}-D\right)\right]
\end{gathered}
$$

where $\mu$ is the Lagrange multiplier associated with the government's budget constraint. Note that (3) applies as long as $D \leq L_{1}$ holds. If $D>L_{1}$, the term $\pi_{1 i} u\left(s_{i}-D\right)$ becomes $\pi_{1 i} u\left(s_{i}-L_{1}\right)$ and the term $(1+\lambda) \pi_{1 i}\left(L_{1}-D\right)$ disappears.

Using the envelope theorem, the FOCs for the policy variables can be written in the following way:

$$
\begin{aligned}
\frac{\partial \mathcal{L}}{\partial \tau}= & -\sum n_{i} u^{\prime}\left(c_{i}\right) y_{i}+\mu \sum n_{i} y_{i}(1-\gamma \tau)=0, \\
\frac{\partial \mathcal{L}}{\partial A}= & \sum n_{i} u^{\prime}\left(c_{i}\right)-\mu=0, \\
\frac{\partial \mathcal{L}}{\partial D}= & -\sum n_{i} \pi_{1 i} u^{\prime}\left(c_{i}^{D_{1}}\right)-\sum n_{i} \pi_{2 i} u^{\prime}\left(c_{i}^{D_{2}}\right) \\
& +\mu \sum n_{i}(1+\lambda) \pi_{1 i}+\mu \sum n_{i}(1+\lambda) \pi_{2 i}=0 .
\end{aligned}
$$

Note that for $D>L_{1}$, the first and third terms disappear from equation (6).

We can then define the following compensated FOCs:

$$
\frac{\partial \mathcal{L}^{c}}{\partial \tau}=\frac{\partial \mathcal{L}}{\partial \tau}+\frac{\partial \mathcal{L}}{\partial A} \frac{d A}{d \tau}=0
$$

and

$$
\frac{\partial \mathcal{L}^{c}}{\partial D}=\frac{\partial \mathcal{L}}{\partial D}+\frac{\partial \mathcal{L}}{\partial A} \frac{d A}{d D}=0
$$

with $\frac{d A}{d \tau}=(1-\gamma \tau) \bar{y}$ and $\frac{d A}{d D}=(1+\lambda) \bar{\pi}_{1}+(1+\lambda) \bar{\pi}_{2}$ derived from the budget constraint, where $\bar{y}=\sum n_{i} y_{i}, \bar{\pi}_{1}=\sum n_{i} \pi_{1 i}$, and $\bar{\pi}_{2}=\sum n_{i} \pi_{2 i}$.

After some manipulations, the compensated FOC for $\tau$ can be written as

$$
\frac{\partial \mathcal{L}^{c}}{\partial \tau}=-\operatorname{cov}\left[u^{\prime}(c), y\right]-\gamma \tau \bar{y} \sum n_{i} u^{\prime}\left(c_{i}\right)=0,
$$

7 We focus on the policy including a demogrant. For comparison, the utilitarian case without a demogrant is provided in section 6.1. 
where $\operatorname{cov}\left[u^{\prime}(c), y\right]=\sum n_{i} u^{\prime}\left(c_{i}\right) y_{i}-\sum n_{i} u^{\prime}\left(c_{i}\right) \bar{y}$. This gives

$$
\tau=\frac{-\operatorname{cov}\left[u^{\prime}(c), y\right]}{\gamma \bar{y} \sum n_{i} u^{\prime}\left(c_{i}\right)}>0 .
$$

The optimal tax rate thus exhibits the usual trade-off between efficiency (the denominator) and redistribution (the numerator, which is positive, since $\operatorname{cov}\left[u^{\prime}(c), y\right]$ is negative).

Similarly, the compensated FOC for $D$ can be written as

$$
\begin{aligned}
\frac{\partial \mathcal{L}^{c}}{\partial D}= & -\operatorname{cov}\left[u^{\prime}\left(c^{D_{1}}\right), \pi_{1}\right]-\operatorname{cov}\left[u^{\prime}\left(c^{D_{2}}\right), \pi_{2}\right] \\
& +\bar{\pi}_{1} \sum n_{i}\left[(1+\lambda) u^{\prime}\left(c_{i}\right)-u^{\prime}\left(c_{i}^{D_{1}}\right)\right] \\
& +\bar{\pi}_{2} \sum n_{i}\left[(1+\lambda) u^{\prime}\left(c_{i}\right)-u^{\prime}\left(c_{i}^{D_{2}}\right)\right]=0,
\end{aligned}
$$

where $\operatorname{cov}\left[u^{\prime}\left(c^{D_{j}}\right), \pi_{j}\right]=\sum n_{i} \pi_{j i} u^{\prime}\left(c_{i}^{D_{j}}\right)-\sum n_{i} u^{\prime}\left(c_{i}^{D_{j}}\right) \bar{\pi}_{j}$, with $j=1,2$.

The compensated derivative $\frac{\partial \mathcal{L}^{c}}{\partial D}$ has four terms (note again that for $D>L_{1}$, the first and third terms will disappear). The last two terms reflect purely the motive of insurance and would be present even if all individuals were identical. The first two terms, on the other hand, reflect the motive of redistribution. Given the assumption that differences in $y$ are sufficiently large compared to differences in dependence probabilities, so that richer individuals save more than poorer ones, we see that the two covariances are positive and thus the first two terms call for a lower deductible. Indeed, since those who are worse off (i.e., the poor) have a higher probability of becoming dependent, transferring resources to the dependence states of nature reinforces redistribution.

It is instructive to study $\frac{\partial \mathcal{L}^{c}}{\partial D}$ by evaluating it at $D=0$ (which means full insurance provided by the government). ${ }^{8}$ It can first be noted that if $\lambda=0$, the last two terms of $\frac{\partial \mathcal{L}^{c}}{\partial D}$ are then equal to zero, which means that, because of the negative first two terms, the compensated derivative is negative, implying that it is optimal to have $D<0$. Thus, if there are no loading costs, the possibility to use insurance for redistribution calls for providing more than full insurance (whereas in the case of identical probabilities, with the covariance terms being equal to zero, full insurance would be optimal under $\lambda=0$ ). On the other hand, if $\lambda>0$, the last two terms are positive at $D=0$, which makes the sign of the whole derivative ambiguous. Indeed, since insurance is costly, it might be no longer optimal to provide more than full, or even just full, insurance. Note,

8 Note that in this case, the assumption about the relative size of differences in $y$ and dependence probabilities is not needed: in the presence of full insurance, wealth levels in the three second-period states of nature are equalized, and differences in dependence probabilities thus play no role in the individual saving decisions. Richer individuals therefore always save more than poorer ones. 
however, that, differently from the case of identical probabilities (where we have less than full insurance as soon as $\lambda>0$ ), full insurance is not necessarily excluded under heterogeneous probabilities and might still be optimal if the loading costs are not too large compared to the redistributional concerns.

To gain a deeper insight into how the optimal deductible is influenced by redistributional concerns, we will now look at the solution obtained under a Rawlsian social welfare function implying the maximization of the least welloff individual's welfare.

\subsection{Rawlsian Case}

The least well-off individual in the considered society is the one having the lowest income and the highest probability of dependence. Let us assume that this individual is of type $i=N$ and, for simplicity, that $y_{N}=0$. Let us also focus on the case of $D \leq L_{1}$ to allow for $D$ being smaller than or equal to zero. The Lagrangian of the government's problem can thus be written as follows:

$$
\begin{aligned}
\mathcal{L}= & u\left(A-s_{N}\right)+\pi_{1 N} u\left(s_{N}-D\right)+\pi_{2 N} u\left(s_{N}-D\right) \\
& +\left(1-\pi_{1 N}-\pi_{2 N}\right) u\left(s_{N}\right) \\
& +\phi\left[\left(1-\frac{\gamma \tau}{2}\right) \tau \bar{y}-A-(1+\lambda) \bar{\pi}_{1}\left(L_{1}-D\right)-(1+\lambda) \bar{\pi}_{2}\left(L_{2}-D\right)\right]
\end{aligned}
$$

where $\phi$ is the Lagrange multiplier associated with the government's budget constraint, and $\bar{y}, \bar{\pi}_{1}$, and $\bar{\pi}_{2}$ are the average values of $y, \pi_{1}$, and $\pi_{2}$ as defined before.

The FOCs for the policy variables can now be written in the following way:

$$
\begin{aligned}
& \frac{\partial \mathcal{L}}{\partial \tau}=\phi \bar{y}(1-\gamma \tau)=0, \\
& \frac{\partial \mathcal{L}}{\partial A}=u^{\prime}\left(c_{N}\right)-\phi=0, \\
& \frac{\partial \mathcal{L}}{\partial D}=-\pi_{1 N} u^{\prime}\left(c_{N}^{D_{1}}\right)-\pi_{2 N} u^{\prime}\left(c_{N}^{D_{2}}\right)+\phi(1+\lambda) \bar{\pi}_{1}+\phi(1+\lambda) \bar{\pi}_{2}=0 .
\end{aligned}
$$

From (9) we have that the optimal tax rate is simply $\tau=\frac{1}{\gamma}$. As far as the optimal deductible is concerned, combining (11) with (10), using (1), and noting that for $D \leq L_{1}$ we have $u^{\prime}\left(c_{N}^{D_{1}}\right)=u^{\prime}\left(c_{N}^{D_{2}}\right)$, we obtain the following FOC:

$$
\begin{aligned}
& (1+\lambda)\left(\bar{\pi}_{1}+\bar{\pi}_{2}\right)\left(1-\pi_{1 N}-\pi_{2 N}\right) u^{\prime}\left(c_{N}^{I}\right) \\
& -\left(\pi_{1 N}+\pi_{2 N}\right) u^{\prime}\left(c_{N}^{D_{1}}\right)\left[1-(1+\lambda)\left(\bar{\pi}_{1}+\bar{\pi}_{2}\right)\right]=0 .
\end{aligned}
$$

It can be easily verified that if $\frac{\pi_{1 N}+\pi_{2 N}}{\bar{\pi}_{1}+\bar{\pi}_{2}}=1+\lambda$, we have $D=0$, and if $\frac{\pi_{1 N}+\pi_{2 N}}{\bar{\pi}_{1}+\bar{\pi}_{2}}>(<) 1+\lambda$, we have $D<(>) 0$. The optimal deductible is thus influenced by the ratio between the sum of the dependence probabilities of the 
poorest individual (which is more generally the poorest individual's probability of becoming dependent, whatever the severity level) and the sum of the population's average dependence probabilities (which is the population's average probability of becoming dependent, whatever the severity level). If the poorest individual's dependence probability is much higher than the population average, it might be optimal to have a negative deductible even in the presence of loading costs. In section 6.2 we show more generally that the optimal deductible decreases when the ratio $\frac{\pi_{1 N}+\pi_{2 N}}{\bar{\pi}_{1}+\bar{\pi}_{2}}$ goes up. The more likely the poorest individual is to become dependent, compared to the average in the society, the more resources need to be transferred to the dependence states of nature.

The main results of this section can be summarized in the following proposition:

Proposition 1 Consider a setting wherein individuals differ in income and dependence probability and wherein a LTC social insurance consists of a deductible and a linear income tax (with a demogrant). A negative correlation between income and risk makes the case for social insurance stronger and may trigger a departure from Arrow's theorem: a zero or even negative deductible may be optimal despite insurance loading costs. This is particularly clear at a Rawlsian optimum, which implies a negative deductible if the ratio between the worst-off individual's and the average dependence probability is greater than one plus the loading cost.

\section{The Case with Private Insurance}

So far we have assumed away the possibility for individuals to purchase insurance on the private market. We are now going to introduce this possibility. Rochet (1991) shows, in the context where both private and social insurance have no loading costs, that a utilitarian optimum implies no use of private insurance as long as there is a negative correlation between individual productivity and the probability of loss. He also shows that private insurance is not used when the government's objective is Rawlsian. We are going to explore if these results are valid in our context.

We therefore assume that there is a market for private LTC insurance and that private insurance can cover part of the social insurance deductible, thus reducing the amount of LTC expenses that the individual effectively incurs. More precisely, we denote by $\alpha_{1 i}\left(0 \leq \alpha_{1 i} \leq 1\right)$ the fraction of the social insurance deductible to be covered in the state of light dependence, and by $\alpha_{2 i}\left(0 \leq \alpha_{2 i} \leq 1\right)$ the fraction to be covered in that of heavy dependence $(i=1, \ldots, N)$. Note that private LTC insurance is possible only when the social insurance deductible is strictly positive (i.e., there is a loss in the dependence 
states of nature); otherwise, no private insurance is provided. We also assume that private insurers face the same loading costs $(\lambda)$ as the government.

The timing we consider is the following. First, the government announces its policy consisting of a linear income tax of rate $\tau$, a demogrant $A$, and social LTC insurance with a deductible $D$. Given this policy, individuals then choose their savings $s_{i}$ and their private insurance coverage characterized by fractions $\alpha_{1 i}$ and $\alpha_{2 i}$ of the social insurance deductible. Reasoning backwards, we will first discuss individual choices and then we will look at the government's policy.

\subsection{Individual Choices}

The expected utility of an individual $i$ can be written as follows:

$$
U_{i}=u\left(c_{i}\right)+\pi_{1 i} u\left(c_{i}^{D_{1}}\right)+\pi_{2 i} u\left(c_{i}^{D_{2}}\right)+\left(1-\pi_{1 i}-\pi_{2 i}\right) u\left(c_{i}^{I}\right),
$$

where $c_{i}=y_{i}(1-\tau)+A-P_{i}-s_{i}$,

$$
c_{i}^{D_{1}}= \begin{cases}s_{i}-\left(1-\alpha_{1 i}\right) D & \text { if } 0<D \leq L_{1}, \\ s_{i}-\left(1-\alpha_{1 i}\right) L_{1} & \text { if } D>L_{1},\end{cases}
$$

$c_{i}^{D_{2}}=s_{i}-\left(1-\alpha_{2 i}\right) D, c_{i}^{I}=s_{i}$, and $P_{i}$ is the private insurance premium given by ${ }^{9}$

$$
P_{i}=(1+\lambda)\left[\pi_{1 i} \alpha_{1 i}+\pi_{2 i} \alpha_{2 i}\right] D
$$

if $0<D \leq L_{1}$, or by

$$
P_{i}=(1+\lambda)\left[\pi_{1 i} \alpha_{1 i} L_{1}+\pi_{2 i} \alpha_{2 i} D\right]
$$

if $D>L_{1}$. The FOC for $s_{i}$ can be written as in (1), whereas the FOCs for $\alpha_{1 i}$ and $\alpha_{2 i}$ are respectively

$$
-u^{\prime}\left(c_{i}\right)(1+\lambda)+u^{\prime}\left(c_{i}^{D_{1}}\right) \leq 0
$$

and

$$
-u^{\prime}\left(c_{i}\right)(1+\lambda)+u^{\prime}\left(c_{i}^{D_{2}}\right) \leq 0 .
$$

Assuming interior solutions and combining (15) and (16), we have $u^{\prime}\left(c_{i}^{D_{1}}\right)=$ $u^{\prime}\left(c_{i}^{D_{2}}\right)$, which implies $\left(1-\alpha_{1 i}\right) D=\left(1-\alpha_{2 i}\right) D\left(\right.$ or $\left.\left(1-\alpha_{1 i}\right) L_{1}=\left(1-\alpha_{2 i}\right) D\right)$.

We can define $M_{i} \equiv\left(1-\alpha_{1 i}\right) D=\left(1-\alpha_{2 i}\right) D$ (or $M_{i} \equiv\left(1-\alpha_{1 i}\right) L_{1}=$ $\left.\left(1-\alpha_{2 i}\right) D\right), M_{i}$ being the true deductible that an individual $i$ has to pay. We can then rewrite the individual problem in terms of $M_{i}$ as follows:

$$
\max _{s_{i}, M_{i}}\left[U_{i}=u\left(c_{i}\right)+\pi_{1 i} u\left(c_{i}^{D_{1}}\right)+\pi_{2 i} u\left(c_{i}^{D_{2}}\right)+\left(1-\pi_{1 i}-\pi_{2 i}\right) u\left(c_{i}^{I}\right)\right],
$$

9 We assume that the private insurers know the individual risk probability. This is quite a standard assumption, made, for instance, in the economics of annuities. 
where $c_{i}=y_{i}(1-\tau)+A-P_{i}-s_{i}, c_{i}^{D_{1}}=c_{i}^{D_{2}}=s_{i}-M_{i}, c_{i}^{I}=s_{i}$, and $P_{i}=(1+\lambda) \pi_{1 i}\left(D-M_{i}\right)+(1+\lambda) \pi_{2 i}\left(D-M_{i}\right)$ if $D \leq L_{1}$, but $P_{i}=(1+\lambda) \pi_{1 i}\left(L_{1}-M_{i}\right)+(1+\lambda) \pi_{2 i}\left(D-M_{i}\right)$ if $D>L_{1}$. The FOC for $s_{i}$ again can be written in the same way as in (1), while the FOC for $M_{i}$ can be written as

$$
u^{\prime}\left(c_{i}\right)\left[(1+\lambda) \pi_{1 i}+(1+\lambda) \pi_{2 i}\right]-\pi_{1 i} u^{\prime}\left(c_{i}^{D_{1}}\right)-\pi_{2 i} u^{\prime}\left(c_{i}^{D_{2}}\right)=0 .
$$

Evaluating the left-hand side of (17) at $M_{i}=0$, it can be easily verified that, as long as $\lambda>0$, the optimal level of $M_{i}$ is always greater than zero. In other words, as long as there are loading costs, private insurance always features a strictly positive deductible (individuals purchase less than full insurance).

For further analysis, it is useful to explore how the optimal level of $M_{i}$ differs between individual types, and in particular how it depends on the two individual characteristics: income and dependence probabilities. In section 6.3, we show that the way in which $M_{i}$ is influenced by these two variables depends on the absolute risk aversion (ARA) exhibited by the utility function. As far as income is concerned, we show that $M_{i}$ is increasing in $y_{i}$ under decreasing absolute risk aversion (DARA), decreasing in $y_{i}$ under increasing absolute risk aversion (IARA), and constant in $y_{i}$ under constant absolute risk aversion (CARA) preferences. ${ }^{10}$ To see the intuition of this result, recall that a higher deductible means less insurance. Since under DARA (under IARA) wealthier people are less (more) risk-averse, they require less (more) insurance. On the other hand, we find that $M_{i}$ is increasing in dependence probability under CARA and IARA preferences, while the effect is ambiguous under DARA. To understand this result, first note that an increase in dependence probability raises the price of insurance. There is then a substitution effect that pushes for buying less insurance (i.e., increasing the deductible). However, there is also a wealth effect in the sense that an increase in the price of insurance makes the individual poorer. In the case of IARA, this translates into the individual becoming less risk-averse, which, like the substitution effect, pushes for a higher deductible. The deductible thus clearly increases under IARA. In contrast, under DARA the wealth effect pushes in the opposite direction to the substitution effect, since poorer individuals are more risk-averse in that case and thus require lower deductibles. The overall effect is thus ambiguous. Finally, under CARA, the wealth effect plays no role and the deductible increases only due to the substitution effect. The results on the effect of income and dependence probability on $M_{i}$ are summarized in table 1 .

Let us now discuss what conclusions can be drawn about the differences in $M_{i}$ between individual types. Under CARA, $M_{i}$ does not depend on income

10 DARA (IARA, CARA) means that absolute risk aversion decreases (increases, remains constant) when wealth increases. For more details, see section 6.3. 
Table 1

Effect of Income and Dependence Probability on $M_{i}$

\begin{tabular}{ccc}
\hline ARA & $\frac{\partial M_{i}}{\partial y_{i}}$ & $\frac{\partial M_{i}}{\partial \pi_{1 i}}\left(\right.$ or $\left.\frac{\partial M_{i}}{\partial \pi_{2 i}}\right)$ \\
\hline DARA & $>0$ & $\lessgtr 0$ \\
\hline IARA & $<0$ & $>0$ \\
\hline CARA & $=0$ & $>0$ \\
\hline
\end{tabular}

but increases with dependence probability, which, taking into account the negative correlation between income and dependence probabilities, implies that poorer (and thus higher-probability) individuals will clearly choose higher deductibles than richer ones. Under IARA, $M_{i}$ also increases with dependence probability and, in addition to this, decreases with increasing income, which again makes it clear that the deductible will be higher for poorer individuals. On the other hand, this is not necessarily the case under DARA. First, under DARA, $M_{i}$ increases with income, which pushes for poorer individuals having lower deductibles. Second, the effect of dependence probability is ambiguous. If it is negative, i.e., if $M_{i}$ decreases with increasing dependence probability, then poorer individuals will indeed have lower deductibles than richer ones. If it is positive, i.e., if $M_{i}$ increases with dependence probability, then the total effect is not clear. No clear-cut comparison can therefore be made in the case of DARA.

\subsection{Public Policy}

We can now turn to public policy. Let us first consider the utilitarian case discussed in section 3.1 but in the presence of the above-described private insurance market. Using the envelope theorem, it can be verified that the FOCs of the social planner's problem are the same as in the case without private insurance (equations (4)-(6)). The compensated FOC for $D$ is thus also the same as equation (7). Let us now analyze this equation, given the presence of private insurance.

When there is little social insurance ( $D$ is high), all individuals buy private insurance (assuming that everyone can afford it) and we have $(1+\lambda) u^{\prime}\left(c_{i}\right)-$ $u^{\prime}\left(c_{i}^{D_{1}}\right)=0$ and $(1+\lambda) u^{\prime}\left(c_{i}\right)-u^{\prime}\left(c_{i}^{D_{2}}\right)=0$ for all $i$. However, this level of $D$ is not optimal, since the compensated derivative is then negative due to the covariance terms. When we decrease the level of $D$, there will be a point where some individuals, those with the highest optimal $M$, will stop buying private insurance. In the cases of CARA and IARA, these will be 
the poorest individuals, while under DARA, that is not necessarily the case. Other individuals, those with lower levels of optimal $M$, will continue insuring themselves on the private market. For these individuals we will thus still have $(1+\lambda) u^{\prime}\left(c_{i}\right)-u^{\prime}\left(c_{i}^{D_{1}}\right)=0$ and $(1+\lambda) u^{\prime}\left(c_{i}\right)-u^{\prime}\left(c_{i}^{D_{2}}\right)=0$, whereas for those who stop buying private insurance we will now have $(1+\lambda) u^{\prime}\left(c_{i}\right)-$ $u^{\prime}\left(c_{i}^{D_{1}}\right)>0$ and $(1+\lambda) u^{\prime}\left(c_{i}\right)-u^{\prime}\left(c_{i}^{D_{2}}\right)>0$. The last two terms of (7) will thus be positive, and this might be the optimal solution if the covariance terms are not too large. On the other hand, it might be optimal to reduce $D$ even more, so that all individuals stop buying private insurance. Thus, we might have the result of no use of private insurance, as in Rochet (1991), but a situation where some individuals insure themselves privately cannot be ruled out either. It is, however, clear that the social optimum implies a nonpurchase of private insurance at least by some individuals in the society (the poorest ones in the cases of CARA and IARA). These individuals get more social insurance than they would purchase on the private market.

The reason why our conclusions differ from those of Rochet (1991) is that we consider a setting where insurance (both social and private) involves loading costs. Indeed, if we assumed, as Rochet (1991), that both social and private insurance were actuarially fair, we would also have a conclusion of no private insurance. To see this, let us suppose for a moment that $\lambda=0$. In that case, the optimal level of $M$ for all individuals is zero (i.e., full insurance). Thus, if the government provides less than full insurance (i.e., $D>0$ ), all the individuals insure themselves privately to reach full insurance. However, this is not optimal from the social point of view, since the last two terms on the left-hand side of (7) are then zero and the first two are negative. Even full social insurance (i.e., $D=0$ ) is not optimal, since the last two terms then remain zero as well. $D$ therefore has to be reduced even more and becomes negative, i.e., more than full insurance is provided. It is clear that then there is no private insurance. $^{11}$

On the other hand, when $\lambda>0$, it might be optimal to have a strictly positive social insurance deductible (i.e., less than full insurance). Moreover, in that case, different individual types require different levels of insurance. Thus, if social insurance is less than full, this might be sufficient for some individual types but insufficient for others, who would then insure themselves on the private market. Note, however, that the case for private insurance becomes weaker if private insurers have higher loading costs than the government.

Let us now look at the Rawlsian case discussed in section 3.2. Again, using the envelope theorem, it can be verified that the FOCs of the social planner's problem have the same form as in the case without private insurance (equa-

11 Note that in Rochet (1991) insurance is not allowed to be more than full, and his result is thus that full public insurance is optimal, which also implies no private insurance. 
tions (9)-(11)). Combining (11) with (10), we have the following FOC for $D$ :

$$
-\pi_{1 N} u^{\prime}\left(c_{N}^{D_{1}}\right)-\pi_{2 N} u^{\prime}\left(c_{N}^{D_{2}}\right)+u^{\prime}\left(c_{N}\right)(1+\lambda)\left(\bar{\pi}_{1}+\bar{\pi}_{2}\right)=0 .
$$

We know from section 3.2 that if $\frac{\pi_{1 N}+\pi_{2 N}}{\bar{\pi}_{1}+\bar{\pi}_{2}}=(>) 1+\lambda$, we have $D=(<) 0$. In these cases, it is clear that there will be no private insurance. On the other hand, if $\frac{\pi_{1 N}+\pi_{2 N}}{\bar{\pi}_{1}+\bar{\pi}_{2}}<1+\lambda$, then $D>0$ and private insurance might occur. As long as $D \geq M_{N}$, equation (17) holds for type $N$ and, using it in (18), we have

$$
u^{\prime}\left(c_{N}\right)(1+\lambda)\left(\bar{\pi}_{1}+\bar{\pi}_{2}-\pi_{1 N}-\pi_{2 N}\right)<0 \text {. }
$$

This means that it is optimal to have $D<M_{N}$. Thus, the worst-off individual will clearly not purchase private insurance. On the other hand, as in the utilitarian case, some other individuals might still find it desirable to insure themselves on the private market.

Proposition 2 summarizes the main insights of this section.

Proposition 2 Introducing the possibility of private insurance with the same loading cost as the social insurance and keeping the setting of Proposition 1, it can be shown that under the utilitarian optimum at least some individuals will not purchase private insurance, but a situation where some other individuals insure themselves privately cannot be excluded. Whether these individuals belong to the top or to the bottom of the income distribution depends on the absolute risk aversion. With the Rawlsian objective, the worst-off individual never purchases private insurance.

\section{Conclusion}

In this paper we have looked at the design of a social insurance for LTC that consists of a linear payroll tax (with a demogrant) and a deductible. We were thus following Arrow's (1963) proposal that an efficient way of providing insurance when there are loading costs is to let the insurees pay all the costs below a given deductible and reimburse them for any expenses above that deductible. We were in particular interested in exploring how the design of such policy is affected by a reasonable assumption that income and the probability of dependence are negatively correlated. In the first part of the paper, we assumed that there was no market for private LTC insurance, whereas we introduced that possibility in the second part.

We show that the presence of a negative correlation between income and dependence probability makes the case for social insurance stronger and might trigger a departure from Arrow's theorem in the sense that, due to redistributional concerns, a zero or even a negative deductible might be optimal despite the presence of loading costs. The influence of redistributional concerns is particularly clearly seen in the case of a Rawlsian social welfare function. In that 
case, a negative deductible becomes optimal as soon as the ratio between the worst-off individual's and the population's average probability of dependence becomes greater than one plus the loading cost.

The introduction of private LTC insurance allows us to compare our results with those of Rochet (1991), who shows, in a context without loading costs, that a negative correlation between individual productivity and the probability of loss implies no use of private insurance. We find that this result does not necessarily hold in our setting involving loading costs. In particular, with a utilitarian social welfare function, we find that the social optimum implies nonpurchase of private insurance at least by some individuals in the society (these are the poorest individuals under CARA and IARA preferences, but not necessarily under DARA), but a situation where some other individuals insure themselves privately cannot be ruled out as long as the optimal social insurance is less than full. With a Rawlsian social welfare function, private insurance is clearly not purchased by the least well-off individual, while it might be purchased by some other ones (but also only if social insurance is less than full).

\section{Appendix}

\subsection{Utilitarian Case without a Demogrant}

Here we consider a more restrictive version of the utilitarian case presented in section 3, namely, a policy in which the government is not able to use a demogrant. The government's problem appears as in section 3.1 except that we now set $A=0$. The FOCs for $\tau$ and $D$ also appear as in (4) and (6). We now define the following compensated FOC:

$$
\frac{\partial \mathcal{L}^{c}}{\partial \tau}=\frac{\partial \mathcal{L}}{\partial \tau}+\frac{\partial \mathcal{L}}{\partial D} \frac{d D}{d \tau}=0
$$

where $\frac{d D}{d \tau}=\frac{(\gamma \tau-1) \bar{y}}{(1+\lambda) \bar{\pi}_{1}+(1+\lambda) \bar{\pi}_{2}}$ is derived from the budget constraint.

After some manipulations, this FOC can be written as

$$
\begin{aligned}
\frac{\partial \mathcal{L}^{c}}{\partial \tau}= & -\left[(1+\lambda) \bar{\pi}_{1}+(1+\lambda) \bar{\pi}_{2}\right] \operatorname{cov}\left[u^{\prime}(c), y\right] \\
& +\bar{y} \operatorname{cov}\left[u^{\prime}\left(c^{D_{1}}\right), \pi_{1}\right]+\bar{y} \operatorname{cov}\left[u^{\prime}\left(c^{D_{2}}\right), \pi_{2}\right] \\
& +\bar{y} \bar{\pi}_{1} \sum n_{i}\left[u^{\prime}\left(c_{i}^{D_{1}}\right)-(1+\lambda) u^{\prime}\left(c_{i}\right)\right] \\
& +\bar{y} \bar{\pi}_{2} \sum n_{i}\left[u^{\prime}\left(c_{i}^{D_{2}}\right)-(1+\lambda) u^{\prime}\left(c_{i}\right)\right] \\
& -\gamma \tau \bar{y}\left[\sum n_{i} \pi_{1 i} u^{\prime}\left(c_{i}^{D_{1}}\right)+\sum n_{i} \pi_{2 i} u^{\prime}\left(c_{i}^{D_{2}}\right)\right]=0 .
\end{aligned}
$$


Note that for $D>L_{1}$, equation (19) is missing the second and fourth terms, as well as the term $(1+\lambda) \bar{\pi}_{1}$ in the first and the term $\sum n_{i} \pi_{1 i} u^{\prime}\left(c_{i}^{D_{1}}\right)$ in the last brackets.

We can then express the optimal tax rate as

$$
\begin{gathered}
-\left[(1+\lambda) \bar{\pi}_{1}+(1+\lambda) \bar{\pi}_{2}\right] \operatorname{cov}\left[u^{\prime}(c), y\right] \\
\tau=\frac{+\bar{y} \operatorname{cov}\left[u^{\prime}\left(c^{D_{1}}\right), \pi_{1}\right]+\bar{y} \operatorname{cov}\left[u^{\prime}\left(c^{D_{2}}\right), \pi_{2}\right]}{\gamma \bar{y}\left[\sum n_{i} \pi_{1 i} u^{\prime}\left(c_{i}^{D_{1}}\right)+\sum n_{i} \pi_{2 i} u^{\prime}\left(c_{i}^{D_{2}}\right)\right]} \\
\bar{y} \bar{\pi}_{1} \sum n_{i}\left[u^{\prime}\left(c_{i}^{D_{1}}\right)-(1+\lambda) u^{\prime}\left(c_{i}\right)\right] \\
+\frac{+\bar{y} \bar{\pi}_{2} \sum n_{i}\left[u^{\prime}\left(c_{i}^{D_{2}}\right)-(1+\lambda) u^{\prime}\left(c_{i}\right)\right]}{\gamma \bar{y}\left[\sum n_{i} \pi_{1 i} u^{\prime}\left(c_{i}^{D_{1}}\right)+\sum n_{i} \pi_{2 i} u^{\prime}\left(c_{i}^{D_{2}}\right)\right]}
\end{gathered}
$$

with the above-mentioned terms disappearing for $D>L_{1}$.

The denominator of (20) is again the efficiency term, which is positive. The numerator, however, unlike in the case with a demogrant, now takes into account not only the motive of redistribution in the first period (the first term, which pushes for a higher tax rate), but also the motives of insurance (the last two terms) and of redistribution in the second period achieved through insurance provision (the second and third terms). As discussed in section 3 , the two covariances entering the second and third terms are positive and call for increasing insurance coverage (i.e., lowering the deductible), which also means increasing the tax rate so that this coverage can be financed.

To gain somewhat more insight, we can look at the compensated FOC $\frac{\partial \mathcal{L}^{c}}{\partial \tau}$ evaluated at $\tau=0$. From the budget constraint, $\tau=0$ obviously implies that no insurance coverage is provided, which in other words means that $D$ is equal to $L_{2}$. Noting that we are now in the case $D>L_{1}$, and recalling the assumption (2), we can write

$$
\begin{aligned}
\left.\frac{\partial \mathcal{L}^{c}}{\partial \tau}\right|_{\tau=0}= & -(1+\lambda) \bar{\pi}_{2} \operatorname{cov}\left[u^{\prime}(c), y\right]+\bar{y} \operatorname{cov}\left[u^{\prime}\left(c^{D_{2}}\right), \pi_{2}\right] \\
& +\bar{y} \bar{\pi}_{2} \sum n_{i}\left[u^{\prime}\left(c_{i}^{D_{2}}\right)-(1+\lambda) u^{\prime}\left(c_{i}\right)\right]>0 .
\end{aligned}
$$

Equation (21) tells us that the optimal tax rate is $\tau>0$, which also implies that the optimal deductible $D$ is lower than $L_{2}$, i.e., it is desirable to provide social LTC insurance. We can also note that the heterogeneity of individuals makes the case for social insurance stronger. Indeed, even if the assumption (2) is not satisfied and the third term of (21) is negative, it could still be possible to have $D<L_{2}$ if the covariance terms are large enough. In other words, even if providing LTC insurance is inefficient from a pure insurance point of view, there may still be a case for social insurance due to 
redistributional concerns. Note also that in this case, without a demogrant, social insurance may be justified even in the absence of a negative correlation between income and dependence probabilities (i.e., with the second covariance equal to zero). ${ }^{12}$ To some extent insurance now also plays the role of a demogrant, since taxes are collected proportionally to income but insurance provision is the same to everyone. Introducing a positive tax and using the proceeds to finance social insurance thus enhances redistribution, as reflected by the first term of (21).

\subsection{Optimal Deductible in the Rawlsian Case}

We are now going to show that the optimal deductible in the Rawlsian case decreases when the probability ratio $\frac{\pi_{1 N}+\pi_{2 N}}{\bar{\pi}_{1}+\bar{\pi}_{2}}$ goes up. To do this, let us first note that the ratio $\frac{\pi_{1 N}+\pi_{2 N}}{\bar{\pi}_{1}+\bar{\pi}_{2}}$ can increase when $\pi_{1 N}$ and/or $\pi_{2 N}$ increases (and the increase in $\bar{\pi}_{1}+\bar{\pi}_{2}$ is sufficiently small) or when $\pi_{1 N}$ and $\pi_{2 N}$ remain the same but the probabilities of other individuals decrease, implying a decrease in $\bar{\pi}_{1}+\bar{\pi}_{2}$. We look at these two cases.

For the first case, we assume for simplicity that $\pi_{1 N}$ increases while $\pi_{2 N}$ and the sum $\bar{\pi}_{1}+\bar{\pi}_{2}$ remain the same (i.e., we assume that the probabilities of some other individuals decrease in such a way that $\bar{\pi}_{1}+\bar{\pi}_{2}$ remains unchanged). We therefore need to verify how the optimal deductible changes due to the increase in $\pi_{1 N}$. From (12) we obtain

$$
\frac{\partial D}{\partial \pi_{1 N}}=\frac{-(1+\lambda)\left(\bar{\pi}_{1}+\bar{\pi}_{2}\right) u^{\prime}\left(c_{N}^{I}\right)+u^{\prime}\left(c_{N}^{D_{1}}\right)\left[(1+\lambda)\left(\bar{\pi}_{1}+\bar{\pi}_{2}\right)-1\right]}{-S O C_{D}}<0,
$$

where $S O C_{D}<0$ is the second-order condition for $D$ and $(1+\lambda)\left(\bar{\pi}_{1}+\bar{\pi}_{2}\right)-$ $1<0$ from the FOC (12).

Turning to the case when $\pi_{1 N}$ and $\pi_{2 N}$ do not change but $\bar{\pi}_{1}+\bar{\pi}_{2}$ decreases, we get

$$
-\frac{\partial D}{\partial\left(\bar{\pi}_{1}+\bar{\pi}_{2}\right)}=\frac{-\left[\begin{array}{c}
(1+\lambda)\left(1-\pi_{1 N}-\pi_{2 N}\right) u^{\prime}\left(c_{N}^{I}\right) \\
+\left(\pi_{1 N}+\pi_{2 N}\right) u^{\prime}\left(c_{N}^{D_{1}}\right)(1+\lambda)
\end{array}\right]}{-S O C_{D}}<0
$$

\subsection{Comparative Statics in the Individual Problem with Private Insurance}

In this subsection, we derive the comparative statics of individual savings $s_{i}$ and the effectively faced deductible $M_{i}$ (chosen simultaneously) with respect

12 This is not true in the case with a demogrant. Indeed, if the assumption (2) does not hold and there is no correlation between income and dependence probabilities, then, evaluating (7) at $D=L_{2}$, we find that decreasing $D$ is never optimal. 
to income $y_{i}$ and the probability of dependence $\pi_{1 i}$ (the case of $\pi_{2 i}$ is analogous).

Fully differentiating (17) and (1) with respect to $y_{i}$, we get respectively

$$
\begin{aligned}
& (1+\lambda)\left(\pi_{1 i}+\pi_{2 i}\right) u^{\prime \prime}\left(c_{i}\right)(1-\tau) \\
& -\frac{\partial s_{i}}{\partial y_{i}}\left[(1+\lambda)\left(\pi_{1 i}+\pi_{2 i}\right) u^{\prime \prime}\left(c_{i}\right)+\pi_{1 i} u^{\prime \prime}\left(c_{i}^{D_{1}}\right)+\pi_{2 i} u^{\prime \prime}\left(c_{i}^{D_{2}}\right)\right] \\
& +\frac{\partial M_{i}}{\partial y_{i}}\left[(1+\lambda)^{2}\left(\pi_{1 i}+\pi_{2 i}\right)^{2} u^{\prime \prime}\left(c_{i}\right)+\pi_{1 i} u^{\prime \prime}\left(c_{i}^{D_{1}}\right)+\pi_{2 i} u^{\prime \prime}\left(c_{i}^{D_{2}}\right)\right]=0
\end{aligned}
$$

and

$$
\begin{aligned}
& \frac{\partial s_{i}}{\partial y_{i}}\left[u^{\prime \prime}\left(c_{i}\right)+\pi_{1 i} u^{\prime \prime}\left(c_{i}^{D_{1}}\right)+\pi_{2 i} u^{\prime \prime}\left(c_{i}^{D_{2}}\right)+\left(1-\pi_{1 i}-\pi_{2 i}\right) u^{\prime \prime}\left(c_{i}^{I}\right)\right] \\
& -\frac{\partial M_{i}}{\partial y_{i}}\left[(1+\lambda)\left(\pi_{1 i}+\pi_{2 i}\right) u^{\prime \prime}\left(c_{i}\right)+\pi_{1 i} u^{\prime \prime}\left(c_{i}^{D_{1}}\right)+\pi_{2 i} u^{\prime \prime}\left(c_{i}^{D_{2}}\right)\right] \\
& -u^{\prime \prime}\left(c_{i}\right)(1-\tau)=0 .
\end{aligned}
$$

For ease of exposition, let us define the following:

$$
\begin{aligned}
& {[1] \equiv\left[(1+\lambda)\left(\pi_{1 i}+\pi_{2 i}\right) u^{\prime \prime}\left(c_{i}\right)+\pi_{1 i} u^{\prime \prime}\left(c_{i}^{D_{1}}\right)+\pi_{2 i} u^{\prime \prime}\left(c_{i}^{D_{2}}\right)\right]<0,} \\
& {[2] \equiv\left[(1+\lambda)^{2}\left(\pi_{1 i}+\pi_{2 i}\right)^{2} u^{\prime \prime}\left(c_{i}\right)+\pi_{1 i} u^{\prime \prime}\left(c_{i}^{D_{1}}\right)+\pi_{2 i} u^{\prime \prime}\left(c_{i}^{D_{2}}\right)\right]<0,} \\
& {[3] \equiv\left[u^{\prime \prime}\left(c_{i}\right)+\pi_{1 i} u^{\prime \prime}\left(c_{i}^{D_{1}}\right)+\pi_{2 i} u^{\prime \prime}\left(c_{i}^{D_{2}}\right)+\left(1-\pi_{1 i}-\pi_{2 i}\right) u^{\prime \prime}\left(c_{i}^{I}\right)\right]<0 .}
\end{aligned}
$$

Solving the system of equations (24) and (25) for $\frac{\partial M_{i}}{\partial y_{i}}$ and $\frac{\partial s_{i}}{\partial y_{i}}$, we obtain

$$
\frac{\partial s_{i}}{\partial y_{i}}=\frac{u^{\prime \prime}\left(c_{i}\right)(1-\tau)\left[[2]-[1] \cdot(1+\lambda)\left(\pi_{1 i}+\pi_{2 i}\right)\right]}{[3] \cdot[2]-[1]^{2}}>0
$$

and

$$
\frac{\partial M_{i}}{\partial y_{i}}=\frac{-u^{\prime \prime}\left(c_{i}\right)(1-\tau) \cdot[4]}{[3] \cdot[2]-[1]^{2}}
$$

where

$$
\begin{aligned}
{[4] \equiv } & {\left[(1+\lambda)\left(\pi_{1 i}+\pi_{2 i}\right)\left(1-\pi_{1 i}-\pi_{2 i}\right) u^{\prime \prime}\left(c_{i}^{I}\right)\right.} \\
& \left.-\left(1-(1+\lambda)\left(\pi_{1 i}+\pi_{2 i}\right)\right)\left(\pi_{1 i} u^{\prime \prime}\left(c_{i}^{D_{1}}\right)+\pi_{2 i} u^{\prime \prime}\left(c_{i}^{D_{2}}\right)\right)\right] .
\end{aligned}
$$

It can be verified that [2] $-[1] \cdot(1+\lambda)\left(\pi_{1 i}+\pi_{2 i}\right)<0$ and [3] $[2]-[1]^{2}>0$, from which the sign of $\frac{\partial s_{i}}{\partial y_{i}}$ then follows immediately. On the other hand, the sign of $\frac{\partial M_{i}}{\partial y_{i}}$ depends on the sign of [4]. The sign of [4] is, however, ambiguous in the general case and depends on the absolute risk aversion (ARA) exhibited by the utility function. In particular, we are now going to show that [4] $>0$ under decreasing absolute risk aversion (DARA), [4] $<0$ under increasing absolute risk aversion (IARA), and [4] $=0$ under constant absolute risk aversion (CARA). 
To see this, let us first note that DARA (IARA, CARA) means that

$$
A R A(c)=\frac{-u^{\prime \prime}(c)}{u^{\prime}(c)}<(>,=) A R A(d)=\frac{-u^{\prime \prime}(d)}{u^{\prime}(d)} \text { for } c>d,
$$

where $\frac{-u^{\prime \prime}(x)}{u^{\prime}(x)}$ is the Arrow-Pratt measure of absolute risk aversion at wealth $x$. Thus, noting that with $M_{i}>0$ we have $c_{i}^{I}>c_{i}^{D_{1}}$, under DARA (IARA, CARA) preferences we can write

$$
\begin{aligned}
& \frac{-u^{\prime \prime}\left(c_{i}^{I}\right)}{u^{\prime}\left(c_{i}^{I}\right)}<(>,=) \frac{-u^{\prime \prime}\left(c_{i}^{D_{1}}\right)}{u^{\prime}\left(c_{i}^{D_{1}}\right)} \\
& \Longleftrightarrow \\
& u^{\prime \prime}\left(c_{i}^{I}\right)>(<,=) \frac{u^{\prime \prime}\left(c_{i}^{D_{1}}\right)}{u^{\prime}\left(c_{i}^{D_{1}}\right)} u^{\prime}\left(c_{i}^{I}\right) .
\end{aligned}
$$

We can then multiply both sides by $(1+\lambda)\left(\pi_{1 i}+\pi_{2 i}\right)\left(1-\pi_{1 i}-\pi_{2 i}\right)$ and subtract from both sides $\left(1-(1+\lambda)\left(\pi_{1 i}+\pi_{2 i}\right)\right)\left(\pi_{1 i} u^{\prime \prime}\left(c_{i}^{D_{1}}\right)+\pi_{2 i} u^{\prime \prime}\left(c_{i}^{D_{2}}\right)\right)$, which gives

$$
\begin{aligned}
& (1+\lambda)\left(\pi_{1 i}+\pi_{2 i}\right)\left(1-\pi_{1 i}-\pi_{2 i}\right) u^{\prime \prime}\left(c_{i}^{I}\right) \\
& -\left(1-(1+\lambda)\left(\pi_{1 i}+\pi_{2 i}\right)\right)\left(\pi_{1 i} u^{\prime \prime}\left(c_{i}^{D_{1}}\right)+\pi_{2 i} u^{\prime \prime}\left(c_{i}^{D_{2}}\right)\right) \\
& >(<,=) \frac{u^{\prime \prime}\left(c_{i}^{D_{1}}\right)}{u^{\prime}\left(c_{i}^{D_{1}}\right)}\left[u^{\prime}\left(c_{i}^{I}\right)(1+\lambda)\left(\pi_{1 i}+\pi_{2 i}\right)\left(1-\pi_{1 i}-\pi_{2 i}\right)\right. \\
& \left.\quad-\left(1-(1+\lambda)\left(\pi_{1 i}+\pi_{2 i}\right)\right)\left(\pi_{1 i} u^{\prime}\left(c_{i}^{D_{1}}\right)+\pi_{2 i} u^{\prime}\left(c_{i}^{D_{2}}\right)\right)\right]=0,
\end{aligned}
$$

where we have used the fact that $c_{i}^{D_{1}}=c_{i}^{D_{2}}$ and that the expression in the last bracket is equal to zero (this follows from combining (17) with (1)).

The left-hand side of the inequality (26) is exactly the definition of [4]; we therefore indeed have, under DARA (IARA, CARA), that $[4]>(<,=) 0$. Coming back to $\frac{\partial M_{i}}{\partial y_{i}}$, we can thus conclude that $\frac{\partial M_{i}}{\partial y_{i}}>(<,=) 0$ with DARA (IARA, CARA) preferences.

Fully differentiating (17) and (1) with respect to $\pi_{1 i}$, we get respectively

$$
\frac{\partial M_{i}}{\partial \pi_{1 i}} \cdot[2]-\frac{\partial s_{i}}{\partial \pi_{1 i}} \cdot[1]-(1+\lambda)^{2}\left(\pi_{1 i}+\pi_{2 i}\right) u^{\prime \prime}\left(c_{i}\right)\left(D-M_{i}\right)=0
$$

and

$$
\frac{\partial s_{i}}{\partial \pi_{1 i}} \cdot[3]-\frac{\partial M_{i}}{\partial \pi_{1 i}} \cdot[1]+(1+\lambda) u^{\prime \prime}\left(c_{i}\right)\left(D-M_{i}\right)+u^{\prime}\left(c_{i}^{D_{1}}\right)-u^{\prime}\left(c_{i}^{I}\right)=0 .
$$

Solving the system of equations (27) and (28) for $\frac{\partial M_{i}}{\partial \pi_{1 i}}$ and $\frac{\partial s_{i}}{\partial \pi_{1 i}}$, we obtain

$$
\begin{aligned}
\frac{\partial s_{i}}{\partial \pi_{1 i}} & =\frac{[2] \cdot\left[u^{\prime}\left(c_{i}^{I}\right)-u^{\prime}\left(c_{i}^{D_{1}}\right)\right]}{[3] \cdot[2]-[1]^{2}}+\frac{(1+\lambda) u^{\prime \prime}\left(c_{i}\right)\left(D-M_{i}\right) \cdot[2] \cdot[4]}{[1] \cdot\left[[3] \cdot[2]-[1]^{2}\right]} \\
& >0(\lessgtr 0)
\end{aligned}
$$


under CARA and IARA (under DARA), and

$$
\frac{\partial M_{i}}{\partial \pi_{1 i}}=\frac{[1] \cdot\left[u^{\prime}\left(c_{i}^{I}\right)-u^{\prime}\left(c_{i}^{D_{1}}\right)\right]}{[3] \cdot[2]-[1]^{2}}+\frac{(1+\lambda) u^{\prime \prime}\left(c_{i}\right)\left(D-M_{i}\right) \cdot[4]}{[3] \cdot[2]-[1]^{2}}>0(\lessgtr 0)
$$

under CARA and IARA (under DARA).

\section{References}

Arrow, K. (1963), Uncertainty and the Welfare Economics of Medical Care, American Economic Review 53, 941-973.

Brown, J. and Finkelstein, A. (2007), Why is the Market for Long-Term Care Insurance So Small?, Journal of Public Economics 91, 1967-1991.

Cremer, H., Pestieau, P., and Roeder K. (2016), Social Long-Term Care Insurance with Two-Sided Altruism, Research in Economics 70, 101-109.

Cremer, H. and Roeder, K. (2013), Long-Term Care Policy, Myopia and Redistribution, Journal of Public Economics 108, 33-43.

Dilnot Commission (2011), Fairer Care Funding: The Report of the Commission on Funding of Care and Support, London: Commission on Funding of Care and Support.

Drèze, J., Pestieau, P., and Schokkaert, E. (2016), Arrow's Theorem of the Deductible and Long-Term Care Insurance, Economics Letters 148, 103-105.

European Commission (2015), The 2015 Ageing Report. Economic and Budgetary Projections for the 28 EU Member States (2013-2060), European Economy 3.

Frank, R. G. (2012), Long-term Care Financing in the United States: Sources and Institutions, Applied Economic Perspectives and Policy 34, 333-345.

Klimaviciute, J. and P. Pestieau (2017), Long-Term Care Social Insurance: How to Avoid Big Losses?, International Tax and Public Finance, forthcoming.

Norton, E. (2000), Long-Term Care, in: Cuyler, A. and J. Newhouse (Eds.), Handbook of Health Economics, Volume 1b, Chapter 17, North-Holland, Amsterdam, 955-994.

Pestieau, P. and Ponthière, G. (2012), Long-Term Care Insurance Puzzle, in: Costa-Font, J. and C. Courbage (Eds.), Financing Long-Term Care in Europe, Palgrave Macmillan, London, 41-52.

Pestieau, P. and Sato, M. (2008), Long-Term Care: the State, the Market and the Family, Economica 75, 435-454.

Rochet, J.-C. (1991), Incentives, Redistribution and Social Insurance, Geneva Papers on Risk and Insurance Theory 16, 143-165. 
Reproduced with permission of copyright owner. Further reproduction prohibited without permission. 